\title{
A Systematic Approach for Closing the Research to Standardization Gap
}

\author{
Bernard Sales, Emmanuel Darmois, Dimitri Papadimitriou, and Didier Bourse \\ Alcatel-Lucent Bell Labs \\ \{bernard.sales, emmanuel.darmois, dimitri.papadimitriou, \\ didier_arnaud.bourse\}@alcatel-lucent.com
}

\begin{abstract}
Standardization activities are recognized as one of the tools to incubate research results and accelerate their transfer to innovative marketable products and services. However, the European Commission (EC) research community and its associated stakeholders acknowledge the lack of research transfer via the standardization channel, generally referred to as the research-tostandardization gap. This chapter analyzes the root causes for this gap and proposes way forward. In particular research-focused standardization is considered as the instrument to address this issue. This chapter shows that prestandardization should be supplemented by a methodology and its associated process aiming to systematically analyze the standardization aspects of research projects and by helping them out to draw their standardization strategy.
\end{abstract}

\section{Introduction}

The Digital Agenda for Europe [1] highlights the importance of ICT standards in delivering interoperability between devices, applications, data repositories, services and networks. It also stresses the fact that standards are to be used strategically as a means of stimulating innovation and promoting interoperability of innovative products.

In this context, the EC has published in June 2011 a series of measures with the objective to have better standards for Europe and to have them faster [2]. As a followup of the publication of the White Paper "Modernising ICT standardization in the EU - The Way Forward" [3] and the related public consultation, one major requirement to strengthen the system of standard-setting in Europe is the recognition that global ICT standards will play a more prominent role in the EU, both from the standardization strategy [4] and regulation standpoints. In particular, regarding EU funded research projects, [4] states, e.g.: "Finally, standards can help to bridge the gap between research and marketable products or services". "A systematic approach to research, innovation and standardisation should be adopted at European and national level to improve the exploitation of research results, help best ideas to reach the market and achieve wide market uptake."

It is well recognized that standards are one important way to promote the translation of research results into practical applications [3] [5] [6] and are also, in 
certain circumstances, the necessary pre-condition for a large deployment and the successful commercialization of a technology. However, research projects do often not engage consistently in standardization because they are not yet convinced by the benefits or/and return on investment of engagement or because they are not familiar enough with their target standardization ecosystem or need guidance on how to address the problem of what to do, where and when to promote their research results in standardization. This lack of engagement is generally referred to as the research-tostandardization gap. The need for practical pre-standardization framework to close this gap is identified as a priority by all stakeholders, including research, ICT industry, EC, but also the Future Internet Assembly (FIA - pre-standardization WG) which has recently proposed a shared action plan to support standardization activities [7] [8]. It is also well accepted that initiatives to better link ICT standardization and ICT R\&D appear to be most effective when carried out at the research planning phase rather than simply at the execution phase of specific research projects [3]. Standardization awareness thus needs to be considered early in the research life cycle and should be an integral part of strategic research agendas.

Starting in Section 2 with an informal survey on research projects requirements, this chapter will analyze the following aspects of the standardization gap: i) what are the root causes of the research-to-standardization gap, ii) how to cope with the specifics of the standardization ecosystem compared to the usual scientific environment and iii) how to satisfy the necessary conditions to efficiently transfer the research results to standardization. For this purpose, Section 3 of this chapter addresses the limits of the classical standardization process in case research results need to be incubated in standardization. In this context, a research-focused standardization phase (generally referred to as pre-standardization), feeding the classical standardization process, needs to be put in place. However, prestandardization needs to be complemented by a methodology and its associated process aiming to systematically analyze the standardization aspects of research projects and by helping them out to draw their strategy. These aspects are discussed in Section 4 of this chapter.

\section{Learning from Research Projects}

In order to start identifying potential expectations and requirements to address the research-to-standardization gap from the research standpoint, an informal set of discussions has been initiated with running Objectives 1.1 projects (UniverSelf, ETICS, EARTH, MEDIEVAL, OneFIT, ...) and specific EC Call 8 proposals under preparation at the time of the writing of this chapter. A first list of requirements has been collected and is presented in Table 1. It should be noted that the requirements are written as received from the survey without any further interpretation. Nevertheless if some of these requirements can not be implemented as such, they translate needs from the research community. 
Table 1. List of Requirements

\begin{tabular}{|c|c|}
\hline Requirements from research project & $\begin{array}{l}\text { Taxonomy/aspect of } \\
\text { the requirements }\end{array}$ \\
\hline $\begin{array}{l}\text { Provide a thorough analysis of standardization bodies candidates and propose } \\
\text { specific steps and community-building activities. }\end{array}$ & Planning \\
\hline $\begin{array}{l}\text { Identify the gaps in current standardization to provide support in coordinating } \\
\text { and informing about potential standardization topics. }\end{array}$ & Planning \\
\hline $\begin{array}{l}\text { Support/guidance during the project submission phase and project } \\
\text { contractualization phase to improve efficiency, coordination, return on } \\
\text { investment and to adapt the standardization plans -based on proposal's } \\
\text { topic(s), EC feedback (hearings/negotiation), reviews, execution phase, } \\
\text { exploitation phase... }\end{array}$ & Planning/Guiding \\
\hline $\begin{array}{l}\text { Provide best current practices on which SDOs are suitable/appropriate targets } \\
\text { for given field of research/standardization, how to approach and provide } \\
\text { consideration in terms of agenda (slow start, SDO processes vs. project } \\
\text { timeframe). }\end{array}$ & Planning/Guiding \\
\hline $\begin{array}{l}\text { Provide networking/connections/know-how/who's doing what on a list of key } \\
\text { standardization topics (and maybe have also special networking events, e.g. } \\
\text { FI weeks, ETSI workshops). }\end{array}$ & Guiding \\
\hline $\begin{array}{l}\text { Document success stories which will be inspiring/leading examples (to } \\
\text { follow/repeat/adapt). }\end{array}$ & Guiding \\
\hline $\begin{array}{l}\text { Make available a single, reference up-to-date knowledge base for current } \\
\text { status of standardization in given topics/areas vs. ending/running projects and } \\
\text { a brief (explanatory) history (why current situation, incl. wrt alternatives). }\end{array}$ & Guiding \\
\hline $\begin{array}{l}\text { Better link the different instruments, e.g. EU-level: Clusters, FIA, projects } \\
\text { and SDOs, as well as at worldwide level incl. North America, Japan/Asia } \\
\text { initiatives/instruments. }\end{array}$ & Linking \\
\hline $\begin{array}{l}\text { Support/help after the end of projects to continue/follow-up initiated } \\
\text { standardization actions. }\end{array}$ & Following-up \\
\hline $\begin{array}{l}\text { Provide up-to-date information with respect to the discussions in the } \\
\text { standardization bodies and support to the submission of contributions from } \\
\text { projects in a coordinated way }\end{array}$ & Mutualizing \\
\hline $\begin{array}{l}\text { Create a platform for joint contributions and submissions to international } \\
\text { standardization bodies. }\end{array}$ & Mutualizing \\
\hline $\begin{array}{l}\text { Set-up of an open forum where participants from different SDO could meet, } \\
\text { avoiding the need for some partner to attend ETSI, IETF, 3GPP meetings to } \\
\text { grasp the key challenges faced by operators and manufacturers. }\end{array}$ & Mutualizing \\
\hline
\end{tabular}

These initial requirements and their taxonomy are a good starting point to frame the discussion on what is needed to address the research-to-standardization gap, for instance:

- Regarding the second requirement in Table 1 , the identification of the gaps should be in close communication with other standardization stakeholders (the industry, regulators, standardization bodies) since researchers on their own are in a bad position to identify the gaps effectively.

- The requirement to "make available a single reference up-to-date knowledge base" seems difficult to achieve but however, [9] provides a first step in this direction.

- The requirement on "support/help after the end of projects to continue/follow-up initiated standardization actions" is really crucial since without such support, standardization plans in the typical short-lived research projects might not be achieved, especially in cases the standardization eco-system is not ready to progress the standardization objectives of the project. 
It is anticipated that the "Planning" and "Guiding" aspects are necessary conditions to reduce the research-to-standardization gap (note that the COPRAS project conducted in the context of FP6 [10] took the same assumptions). On the other hand, the "Linking", "Following-up" and "Mutualizing" aspects provide means to support more efficiently the pre-standardization actions. As one of the objectives is to address the root causes of the research-to-standardization gap, the focus of this chapter is placed on "Planning" and on "Guiding" while the "Linking", "Following-up" and "Mutualizing" aspects will no longer be discussed in this chapter.

\section{Standardization and Pre-standardization}

Standardization of protocols and interfaces has played and is still playing a key role in the Internet development. In particular, the IETF has imposed itself as the main Internet protocols factory while other standardization bodies like IEEE, ITU-T, 3GPP and $\mathrm{W} 3 \mathrm{C}$ are standardizing the infrastructure and technology enablers creating the necessary open ecosystem that contributed to the Internet development.

However, the work in standardization is dwindled by its participant strategy in terms of $\mathrm{R} \& \mathrm{D}$ and conflicting business objectives leaving in practice a very little window to the research and academia communities to influence the process. One could observe that in the early days of the Internet, its standardization was driven by the research community. This materialized by the creation of the IETF that was an emanation from the research community. Over time, as the Internet and its associated technologies progressively matured and were deployed at a larger scale, the Internet standardization gradually shifted to engineering and operational problems (the IETF is often qualified today as "problem-driven"). As a result, even though the research community is still involved in the Internet standardization process, its influence is eroding over time. Nevertheless, the involvement of the research community in standardization can bring a lot of added value to the industry (in particular when practical use cases are identified at this stage of the process) since it allows early derisking of disruptive ideas by confronting them to i) executability/developability, ii) deployability, and iii) market environment and, if successful, will accelerate penetration of those innovative ideas.

In this context, a research-focused standardization phase needs to complement the classical standardization process. In this model, the research-focused standardization phase will feed the classical standardization process with a stream of de-risked ideas that will, if successful, lead to a full standardized solution. It has to be noted that the interactions and discussions in the context of pre-standardization can also directly feed back the research project with valuable inputs to be further considered inside the project ("external loop"). For this reason, this phase intends to bridge the research-tostandardization gap and is generally referred to as the pre-standardization phase.

Major standardization bodies are adapting their processes to capture these requirements. For instance, ISOC created in the 90's the IRTF (the research arm of the IETF), the ITU-T defined the concept of Focus Group, the IEEE established IEEE-SA Industry Connections Program and the W3C the W3C Incubator Activity. 
In 2006, ETSI defined the concept of Industry Specification Group (ISG). All these pre-standardization processes share the same principles: they are open to academia and are based on a lightweight procedural structure compared to their "mother" standardization groups. On the other hand, one can observe that, in the context of the Internet these pre-standardization structures are not yet used at their full potential. In particular, when "pre-standardization" processes/organizations exist, they have often evolved in two directions, either by focusing on shorter-term engineering problems the standardization body is recognized for (and, in turn, being perceived as no longer fulfilling a research role) or by focusing on longer-term architectural problems (and, in turn, being perceived as disconnected from the rest of the standardization organization activities). It is also anticipated that the results of the Future Internet and Future Networks research will have the potential to boost the volume of prestandardization activities and could really lead to the launching of the Future Internet pre-standardization process.

It should be noted that not all research results need to be incubated in prestandardization. Depending on the standardization lifecycle and rationality, research results can go directly to the classical standardization regime without going through a preliminary pre-standardization phase.

For instance, the classical standardization regime is not yet ready to standardize all aspects related to Self Managed Networks and, as a result, pre-standardization is required (e.g. in an ETSI ISG or in an IRTF Research Group). In contrast, regarding Carrier Ethernet, the standardization regime is mature; there is no need to go through a pre-standardization phase.

Pre-standardization is the necessary tool helping create an environment that is, when required, more suitable to incubate research ideas than the classical standardization regime. Despite its great potential, pre-standardization alone (i.e. without a built-in link to standardization and without a framework to systematically analyze the standardization aspects of research projects and helping them out to draw their strategy) is not broad enough to motivate researchers to present and defend their ideas only there.

\section{Methodological Aspects}

\subsection{The Need for Standardization Strategy}

According to the experience acquired over years by the co-authors of this chapter, in order to be really effective, standardization actions should be defined from and supported by a well defined standardization strategy/planning. In the context of this chapter, a standardization strategy is defined as a path of standardization-related actions and objectives (in a few complex cases, a strategy may even comprise parallel paths). Without any standardization strategy, the standardization actions are in general unsuccessful or lead to suboptimal results. In the worst case, the standardization achievements may even be conflicting with the research objectives of the project. 
As a result, the standardization strategy has to be carefully addressed and has to consider multiple dimensions including the maturity of the standardization ecosystem, the position of the technology proposed for standard in the standardization life-cycle, the objectives of the research projects, the possible open issues and the research project maturity. To deal with the dynamics of both standardization and research environments, the strategy needs to be reassessed on a regular basis. As a consequence, this process is characterized by iterative cycles of defining/refining the strategy, adapting actions and expected achievements in standardization bodies.

To help and guide research projects, a methodology to analyze the standardization aspects of research projects and the associated process (mechanisms) need to be further developed. The combination of the proposed methodology and its associated process will enable the research projects to define and reassess their standardization strategy that is a necessary condition to address the researchto-standardization gap.

\subsection{A Systematic Way to Draw the Strategy}

A systematic methodology to analyze the standardization aspects of a research project and to draw its standardization strategy/approach was already proposed by the authors of this chapter [11] [12]:

- Step 1: Identify what needs to be standardized (interfaces, etc) to allow the technology proposed by the project to be interoperable and deployable at large scale. In general, this step implies the identification of an "initial" architecture.

- Step 2: Identify the role and impacts of standardization bodies on the business segment targeted by the project. At this step, standardization bodies are categorized as fulfilling a role in the standardization food chain, i.e. requirements, architecture, solution/protocol/interface and interoperability/testing.

- Step 3: Evaluate the need to improve the standardization eco-system to maximize the chance of success, this can materialize either by creating new (pre-) standardization technical committee and/or by attracting major stakeholders.

- Step 4: Identify the "structuring" dimensions (i.e. what characterizes the standardization objectives trajectory/path) for the proposed technology/system to define a) the criteria to shape the associated standardization target(s) of the research projects b) the necessary conditions to meet in order for the technology/system to enable its standardization. The output of this step is a standardization objectives trajectory to be realized.

The main objective of the methodology is to guide research projects in identifying their standardization needs and approach in a systematic way to ensure that all the necessary aspects are analyzed and developed. The methodology can lead to three types of results: 
1. The first one is when standardization is not needed at all and when the lack of standardization is not a roadblock for the large scale deployment of the technology being designed by the research project.

2. The second case is when standardization is required but the related standardization ecosystem is not ready/in place to progress the standardization objectives of the project. In other words, it is very unlikely that a standardization body will accept to incorporate this necessary work items in its standardization work program. In this case, the technology needs to be incubated in a (pre-)standardization group. In general, this will require the creation of a new (pre-)standardization WG.

3. The latter case is when the technology can be directly pushed in standardization bodies without the need to go through a pre-standardization phase.

In general, Step 1 of the methodology is conducted in an analytical way. In this context, having an (initial) architecture is of a great benefit since it will enable to systematically enumerate all the interfaces and to analyze formally which of them needs to be standardized to enable the further transfer of the technology/system to marketable products and/or services. This analytical study can be complemented by an experimental facility/test-bed whose objectives, when affordable, are to benchmark components, to identify their behavior in large-scale setup, and to detect non interoperable components/features that will at the end require some form of standardization. It should be noted that this four steps methodology has been already successfully applied to several Alcatel-Lucent small and medium size research projects. This methodology was also used to define the standardization plan of some EC FP7 Future Internet research projects. This contributes to validate the applicability of the methodology but does not demonstrate that the methodology can be deployed at the large scale (e.g. at the FIA level).

\subsection{Application of the Four Steps Methodology to ECODE Project}

FP7-ICT 2007 ECODE project (Grant 223936) [13] is the first FP7 project to which the above methodology was used.

The objective of the ECODE project was to associate new architectural network components, based on machine learning principles, architectures and techniques in networking platforms to assist operation (automated, on-line analysis), improve performance gain by predicting and adapting decisions, and extend Internet functionality (e.g., diagnostics, network intrusion/attack detection, etc.) [14].

The four-step methodology was applied at the time of writing the project proposal; the application of the methodology was employed to document the standardization part of the project proposal. The results of the initial application of the four steps of the methodology was summarized in the form of a dashboard [12] that was used at different stages of the project (see Figure 1). 


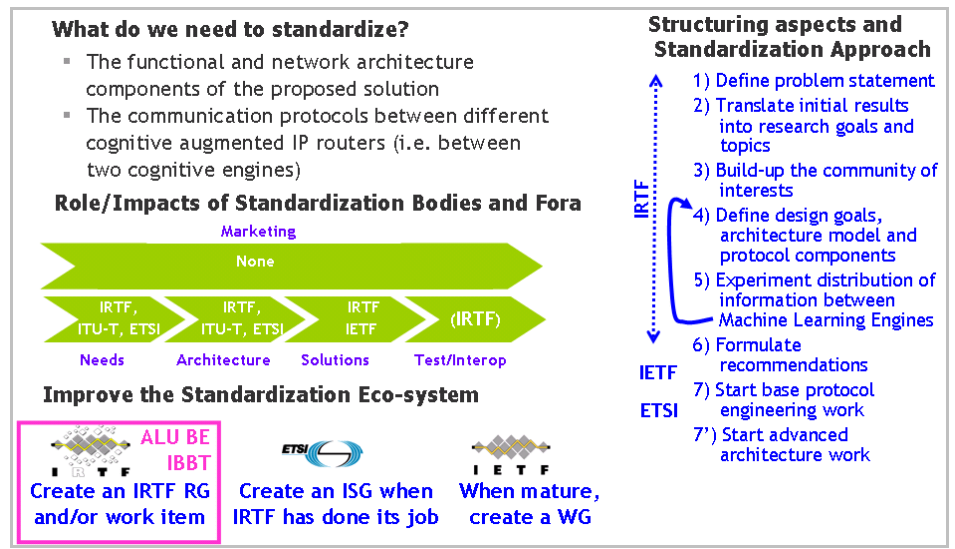

Fig. 1. Standardization Dashboard for Research Projects

In this context, Step 4 of the methodology enabled the ECODE team to link their envisioned usage scenarios for their technology (identified as structuring aspects by the methodology) with specific standardization objectives. In particular by applying the step 4 of the methodology, the ECODE project has identified two objectives for the introduction of machine learning component (the above-defined 'structuring' aspects): 1) address current Internet operational challenges; 2) further extend Internet functionalities (diagnosability, security, etc.).

From the standardization perspective, the first objective implies that protocols must be standardized in the IETF, while the second one implies that an advanced architecture should be defined, e.g. in ETSI. In addition, as machine learning techniques were never used before in the context of Internet and are challenging in the context of the Internet deployment, it would be necessary to have a prestandardization phase, e.g. in the IRTF (see the dashboard in Figure 1).

Using the proposed methodology, the standardization strategy was reassessed twice in the course of the project due among others to the change in IRTF priorities. This reassessment helped the ECODE partners in determining their standardization plan beyond the lifetime of the project. All these steps enabled the ECODE project to define and refine systematically a coherent standardization strategy starting from requirements, followed by the identification of the target standardization bodies and roles and ending with the definition of the standardization approach and objectives.

\subsection{Implementing the Methodology at Large Scale}

Having outlined a methodology to assist the research community to identify the standards needs, approach and objectives associated to a research project, the "process" aspects (the mechanics) that will enable the implementation and validation of the methodology on a large scale (i.e. at the FIA level) have to be addressed. 
For this purpose, the relation between research projects and their standardization ecosystem are analyzed in terms of downstream and upstream channels. The downstream channel is materialized by the participation and contributive efforts of research experts to the standardization bodies: participation to meetings, submission and presentation of contributions and leadership positions when appropriate. This downstream channel is generally managed by the research project, resulting in a standardization approach defined at the project level as part of the dissemination and exploitation plans. However, as already mentioned before, researchers are not necessarily attracted by or familiar with the targeted standardization environment. There are multiple reasons that can explain this:

1. Research project objectives are research results-driven whereas standardization objectives are engineering consensus-driven.

2. Participation to most standardization bodies requires an annual fee. Unless that cost can be sustained by academic and research institutes, the research project cannot access the standardization organizations working documents (contributions, meeting minutes, etc.);

3. Standardization debates and positioning of actors are often driven by "economical" interests beyond any possible influence of academics and research institutes (not recognized as full-fledged players).

4. Each standardization body operates with its own specific methods and procedures whilst research projects standardization plans require combining actions in multiple standardization bodies - which in turn increase the complexity for the research project to conduct its standardization actions.

As a result, the standardization strategies and plans of a research project are often defined on an ad-hoc basis and sometimes, even misleading and/or incomplete. When a project has an insufficient understanding of the standardization environment, it may opt for easily implementable workarounds. For instance, its contributions are submitted only once to a standardization organization and sometimes not presented in meetings. In this case, the standardization body just "notes" that the contribution was submitted and, as a result, the technology designed by the research project will never lead to a standard. Moreover, contributions from research projects are also often missing their target: expecting that the outcomes of research as reported in project deliverables will be accounted for as-is by the targeted standardization organization is not realistic. Two main causes for failure can be identified: i) lack of adoption of the conventions and writing style of the targeted standardization body, and ii) difficulty to confront its output with various technical communities (system engineers, network engineers, operation, etc.) before it can have a technological impact on the course of the standards making.

In addition to the 'downstream' channel, there is also an 'upstream channel' from the standardization community to the research projects. In the simplest way currently available, this corresponds to the information published by standardization organizations on their web sites. This information is often general purpose and as such not targeted and/or tailored to/for the research community; it is at best informative but often rather useless for researchers. As noted, if project partners do 
not pay the standards organization membership fees (when applicable), this information is not even accessible at all (e.g., for copyright reasons). In some cases, this upstream channel is better managed when standardization bodies organize 'research to standardization' workshops (e.g. [5], [6]) though, often, the audience on these workshops is composed of the research experts already involved in the standardization work.

It is postulated by the authors of this chapter that three conditions need to be satisfied in order to improve the quality of the downstream (from research to standardization) channel and maximize the value of the output: 1) availability of information from standardization bodies that is directly relevant to the research project; 2) mutual understanding, at both ends of the channel, that research results have reasonable chances to be adopted in the appropriate standardization context; and 3) joint determination of the trajectory (sequence of standardization actions with starting and ending points) by means of a standardization strategy.

To satisfy the three conditions to improve the downstream (from research to standardization) channel, the upstream (from standardization to research) channel needs to be enhanced in the following ways:

1. Provide information related to standardization status and evolution specifically targeted to the research community. (A first step in this direction is the information repository provided by the FIA pre-standardization WG [9]) A criterion of success for this approach is the initiation, within a standardization organization that takes this path, of a standardization track that was not previously addressed. Two cases shall be however distinguished. In the case of a standardization organization already working on the technology to which the research project contributes, it is less complex to put in place the process, but the impact on the technology specification will probably be smaller. When the standardization organization is not yet working on the new technology proposed by the research project, more effort will be required but in case of success - impact will be greater since it will define a new technology specification track.

2. Proactively support the research project by a team of dedicated experts with a strong 'research and standardization' background. The role of these experts, the 'Research-to-Standards' team, is i) to guide the research projects on the definition of their standardization strategy (using the methodology defined in Section 6) including the sequence of standardization actions required to ensure that the technology under consideration will be developable and deployable at a large scale (necessary condition), and ii) to regularly follow-up with research teams on progress and open issues and/or blocking factors, to help progressing on the trajectory and propose possible remediation actions in case of problem.

3. Research projects must be convinced of the benefits to use a well defined methodology to define their standardization strategy and trained on how to use the methodology.

In the context of autonomic networking (e.g. see [15]), the downstream channel from research projects to standardization is currently working quite well e.g. in terms of i) 
number of contributions, ii) stepwise approach based on architecture - uses cases solutions, iii) improvement of the (pre-)standardization infrastructure with the creation of the ETSI ISG on Autonomic network engineering for the self-managing Future Internet (AFI), iv) reach in terms of standardization bodies, etc. The only issue is the critical mass, i.e. only few FP7 research projects dealing with autonomic networking are involved in the (pre-)standardization process. If someone had implemented the improvements proposed in this Chapter to the upstream channel from standardization to the research projects dealing with autonomic networking, the expected results would have been to embark in the standardization effort almost all the running FP7 research projects relevant in this context (and even EUREKA and National projects in case of full and well organized implementation of the proposed process).

Regarding the ECODE project discussed in section 5.2, the downstream channel was not working as expected (i.e. the creation of an IRTF Research Group), but this is mainly due to the change of priorities in the targeted pre-standardization body. However, the enhancements to the upstream channel as proposed in this chapter enable the project to adapt their standardization strategy to cope with this situation.

Future work will consist in applying the methodology on a set of representative research projects in order to characterize the expected benefits and give more guidelines and cook book on its implementation.

\section{Conclusion}

Research-focus standardization (in general referred to as "pre-standardization") is a necessary instrument to attract a critical mass of researchers to participate in standardization process. But this instrument alone is not sufficient. Actually prestandardization should be supplemented by a dedicated planning effort at the project research level that will have to be materialized in a well defined standardization strategy. However, standardization body operates with its own specific methods and procedures. In addition, the necessary research projects standardization actions require combining actions in multiple standardization bodies which in turn increase the complexity for the research project to define its standardization strategy. As a way to guide the research projects, the authors provide a methodology and its associated process aiming to systematically analyze the standardization aspects of a project and by helping them out to draw their strategy.

The above enhancements can be either implemented by key representative standardization organizations or implemented by an entity external to standardization bodies (but closely linked/interacting with the key standardization organizations). To adopt these enhancements, standardization bodies must be convinced of the usefulness of the approach before engaging resources to implement the proposed process. It is currently difficult to anticipate the benefits of having this process implemented in key standardization organizations or in an entity outside the standardization bodies. Even more important, research projects must be convinced of the benefits to use a well defined methodology to define their standardization strategy and should be trained on how to use the methodology. The authors believe that the 
proposed process, once validated in the Future Internet context, e.g. on a selected representative research projects, can be deployed at a large scale and deliver the expected benefits to research and standardization.

Open Access. This article is distributed under the terms of the Creative Commons Attribution Noncommercial License which permits any noncommercial use, distribution, and reproduction in any medium, provided the original author(s) and source are credited.

\section{References}

1. European Commission, A Digital Agenda for Europe, COM(2010) 245 final/2, Brussels (August 26, 2010)

2. European standardisation policy, http://ec.europa.eu/enterprise/ policies/european-standards/standardisation-policy/index_en. htm

3. European Commission, Modernising ICT Standardisation in the EU: the Way Forward, COM(2009) 324, Brussels, July 3 (2009)

4. European Commission, A strategic vision for European standards: Moving forward to enhance and accelerate the sustainable growth of the European economy by 2020, COM 2011, 31, Brussels, June 1 (2011)

5. ETSI, Future Network Technologies Workshop, Sophia Antipolis (March 10-11, 2010), http://www.etsi.org/WebSite/NewsandEvents/Past_Events/201_Fu tureNetworkTechnologies_WS.aspx

6. ETSI, 2nd ETSI Workshop on Future Networks Technologies, Sophia Antipolis (September 26-27, 2011), http://www.etsi.org/WebSite/NewsandEvents / Past_Events/2010_FutureNetworkTechnologies_WS.aspx

7. Bourse, D.: Future Internet Assembly (FIA) Pre-standardisation WG - Review of Objectives and Progresses, Poznan FIA, Poznan (October 25, 2011)

8. FIA Standardisation Support, http://fisa.future-internet.eu/index.php/ FIA_Standardisation_Support

9. Pre-standardisation activities in FIA, http://fisa.future-internet.eu/ index.php/Pre-standardisation_activities_in_FIA

10. COPRAS project, http: / / www.w3 .org/2004/copras /

11. Papadimitriou, D., Sales, B.: A path towards strong architectural foundation for the internet design. In: 2nd Future Network Technologies Workshop, ETSI, Sophia Antipolis, France, September 26-27 (2011)

12. Papadimitriou, D., Sales, B.: Cognitive Augmented Routing System and its Standardisation Path. In: Future Network Technologies Workshop, ETSI, Sophia Antipolis, France, March 10-11 (2010)

13. ECODE (Experimental COgnitive Distributed Engine) project, http://www . ecodeproject.eu/

14. Papadimitriou, D., Donnet, B.: A Cognitive Routing System for the Internet. In: 8th Würzburg Workshop on IP (Euroview 2008): Joint EuroNF, ITC, and ITG Workshop on Visions of Future Generation Networks, Würzburg, Germany, July 21-22 (2008)

15. Ciavaglia, L., Altman, Z., Patouni, E., Kaloxylos, A., Alonistioti, N., Tsagkaris, K., Vlacheas, P., Demestichas, P.: Coordination of Self-Organizing Network Mechanisms: Framework and Enablers. In: Proceedings of the Special Session on Future Research Directions at ICST MONAMI 2011 Conference, Aveiro, Portugal (September 2011) 